\title{
Satellite and Opacity Effects on Resonance Line Shapes Produced from Short-pulse Laser Heated Foils
}

R. Shepherd, P. Audeburt, H.-K. Chen, K. B. Fournier, O. Peyreusse, S. Moon, R. W. Lee, D. Price, L. Klein, J. C. Gauthier, P. Springer

U.S. Department of Energy

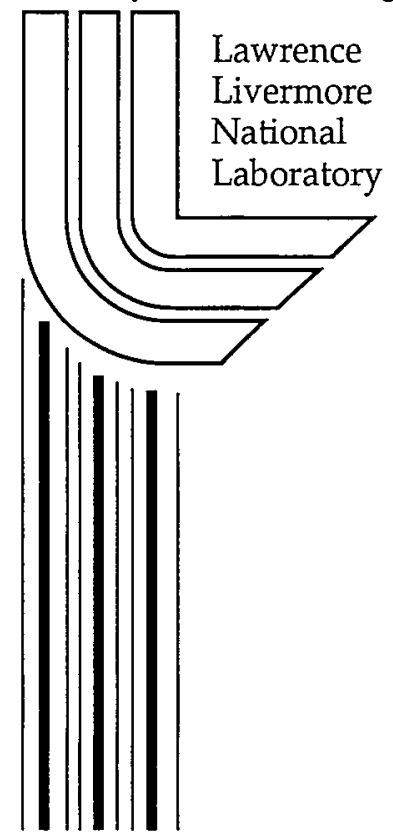

This article was submitted to

Radiative Properties of Hot Dense Matter, Saint-Malo, France,

September 16-20, 2002

December 3, 2002 


\section{DISCLAIMER}

This document was prepared as an account of work sponsored by an agency of the United States Government. Neither the United States Government nor the University of California nor any of their employees, makes any warranty, express or implied, or assumes any legal liability or responsibility for the accuracy, completeness, or usefulness of any information, apparatus, product, or process disclosed, or represents that its use would not infringe privately owned rights. Reference herein to any specific commercial product, process, or service by trade name, trademark, manufacturer, or otherwise, does not necessarily constitute or imply its endorsement, recommendation, or favoring by the United States Government or the University of California. The views and opinions of authors expressed herein do not necessarily state or reflect those of the United States Government or the University of California, and shall not be used for advertising or product endorsement purposes.

This is a preprint of a paper intended for publication in a journal or proceedings. Since changes may be made before publication, this preprint is made available with the understanding that it will not be cited or reproduced without the permission of the author.

This work was performed under the auspices of the United States Department of Energy by the University of California, Lawrence Livermore National Laboratory under contract No. W-7405-Eng-48.

This report has been reproduced directly from the best available copy.

Available electronically at http://www.doc.gov/bridge

Available for a processing fee to U.S. Department of Energy

And its contractors in paper from

U.S. Department of Energy

Office of Scientific and Technical Information

P.O. Box 62

Oak Ridge, TN 37831-0062

Telephone: (865) 576-8401

Facsimile: (865) 576-5728

E-mail: reports@adonis.osti.gov

Available for the sale to the public from

U.S. Department of Commerce

National Technical Information Service

5285 Port Royal Road

Springfield, VA 22161

Telephone: (800) 553-6847

Facsimile: (703) 605-6900

E-mail: orders@ntis.fedworld.gov

Online ordering: http://www.ntis.gov/ordering.htm

OR

Lawrence Livermore National Laboratory

Technical Information Department's Digital Library

http://www.llnl.gov/tid/Library.html 


\title{
Satellite and opacity effects on resonance line shapes produced from short-pulse laser heated foils
}

\author{
Ronnie Shepherd ${ }^{\mathrm{a}, *}$, P. Audebert ${ }^{\mathrm{b}}, \mathrm{H}-\mathrm{K}$. Chen ${ }^{\mathrm{a}}$, \\ K. B. Fournier ${ }^{\text {a }}$, O. Peyreusse ${ }^{c}, S$. Moon $^{a}$, R. W. Lee ${ }^{a}$, \\ D. Price ${ }^{a}$, L. Klein ${ }^{d}$, J.C. Gauthier ${ }^{b}$, P. Springer ${ }^{a}$ \\ ${ }^{a}$ Physics and Advanced Technologies, \\ Lawrence Livermore National Laboratory, \\ P.O. 808, Livermore Ca., USA,94550. \\ ${ }^{\mathrm{b}}$ Laboratoire pour l'Utilisation des Lasers Intenses, UMR7605, \\ CNRS - CEA - Universıté Paris VI - École polytechnique, \\ 91128 Palaiseau, France \\ ${ }^{\circ} C E A-D I F$, Bruyère-le-Châtel, France \\ 'Physics and Astronomy Department, \\ Howard University, Washıngton D.C., USA
}

\begin{abstract}
We measure the He-like, time-resolved emission from thin foils consisting of $250 \AA$ of carbon-250 $\AA$ of aluminum and $500 \AA$ aluminum illuminated with a 150 fs laser pulse at an intensity of $1 \times 10^{19} \mathrm{~W} / \mathrm{cm}^{2}$. Dielectronic satellite contributions to the $1 s^{2}-1 s 2 p\left({ }^{1} \mathrm{P}\right), 1 s^{2}-1 \mathrm{~s} 3 \mathrm{p}\left({ }^{1} \mathrm{P}\right)$, and $1 \mathrm{~s}^{2}-1 \mathrm{~s} 4 \mathrm{p}\left({ }^{1} \mathrm{P}\right)$ line intensities are modeled using the configuration averaged code AVERROĖS and is found to be significant for all three resonance lines. The contribution of opacity broadening is inferred from the data and found to be significant only in the $1 s^{2}-1 s 2 p\left({ }^{1} \mathrm{P}\right)$.
\end{abstract}

Key words: Short pulse laser produced plasma, x-ray spectroscopy, short pulse laser

* Corresponding author. shepherd1@llnl.gov 


\section{Introduction}

There has long been a debate in plasma spectroscopy on the ability (and some would say the existance) of line shifts caused by the fields produced by ions at high particle density[1-4]. The line positions are most significantly altered in plasmas when the kinetic energy is comparable to the kinetic energy of the particles, i.e., large Coulomb gammas $\left(\Gamma_{22}, \equiv \bar{Z}^{2} e^{2} /\left(\frac{3 N_{1}}{4 \pi}\right)^{1 / 3} K_{B} T \geq 1\right)$. Recent results reported in the literiture have lead to new questions about the ability to measure polarization effects on spectral lines[5]. These experiments have been performed on thin layers buried in solid targets heated with high intensity ( $>1 \times 10^{17} \mathrm{~W} \mathrm{~cm}^{2}$ ), ultrashort laser pulses ( $\leq 150 \mathrm{fs}$ ). At issue is the ability to extract accurate resonance line position data from plasmas that are extremely dynamic, a source of large numbers of satellites, and could have optical depth issues.

To illuminate the subject, we have studied K-shell aluminum emission spectra from thin foils heated with an ultrashort pulse laser by heat conduction. A thin foil consisting of $250 \AA$ of carbon and $250 \AA$ of aluminum is illuminated with a 150 fs laser pulse at an intensity of $1 \times 10^{19} \mathrm{~W} / \mathrm{cm}^{2}$. This data is compared to $\mathrm{x}$-ray spectra from a $500 \AA \mathrm{Al}$ foil $(500 \mathrm{Al})$. The foil illuminated on the carbon side $(250 \mathrm{C} / \mathrm{Al})$ does not heat the aluminum directly by laser but instead by heat conduction. After heating, minimal radiation cooling and longitudinal heat conduction occur due to the targets low atomic number and thickness, allowing hydrodynamic expansion to dominate the cooling process. The resulting plasma is extremely unique and dynamic. The plasma conditions early in the foils evolution is believed to consist of strongly coupled ions $\left(\Gamma_{z \imath}>2\right)$ submerged in a weakly coupled electron bath $\left(\Gamma_{e e} \leq 0.02\right)$. As the foil cools, the recombination lags the decrease in electron temperature and the average ionization remains high, increasing the particle correlations with time. This provides a unique environment to study line shapes. The time resolved $1 \mathrm{~s}^{2}-1 \mathrm{~s} 2 \mathrm{p}\left({ }^{1} \mathrm{P}\right)\left(\mathrm{He}_{\alpha}\right), 1 \mathrm{~s}^{2}-1 \mathrm{~s} 3 \mathrm{p}\left({ }^{1} \mathrm{P}\right)\left(\mathrm{He}_{\beta}\right)$, and $1 \mathrm{~s}^{2}-1 \mathrm{~s} 4 \mathrm{p}\left({ }^{1} \mathrm{P}\right)\left(\mathrm{He}_{\gamma}\right)$ spectra is collected with a 500 fs x-ray steak camera mated to a two crystal von Hamos spectrograph. Additionally, these data are compared to data from the same target heated from the aluminum side $(250 \mathrm{Al} / \mathrm{C})$ and a $500 \AA$ aluminum foil $(500 \mathrm{Al})$ target illuminated at the same laser intensity. The line shapes are shown to be strongly influenced by satellite emission and opacity. A quantitative measurement of the line center opacity of the $\mathrm{He}_{\alpha}$ is made by matching the optically thin Li-like satellite emission intensity from the $250 \mathrm{C} / \mathrm{Al}$ case and the $500 \mathrm{Al}$ case and inferring the peak line cneter emission intensity from the $500 \mathrm{Al} \mathrm{He}$. 


\section{Experimental Setup}

We performed our experiment at the Lawrence Livermore National Laboratory UltraShort Pulse laser facility. The laser has been described in detail elsewhere so only a brief description is given here [6]. The laser utilizes chirped pulse amplification to generate high energy, 150 fs pulses. The pulses from an $82 \mathrm{MHz}$ Ti:Sapphire oscillator are temporally stretched, amplified then recompressed in a vacuum compressor. After compression, the pulse is frequency doubled with a $1.5 \mathrm{~mm}$ thick $\mathrm{KD}^{*} \mathrm{P}$ crystal and focussed on the target with an $\mathrm{f} / 3.5$ off axis parabola. The measured energy on target is 150 to $200 \mathrm{~mJ}$ at $400 \mathrm{~nm}$ and is focusable to a FWHM spot size of $2 \mu \mathrm{m}$.

Pulses out of the oscillator deviating more than $10 \%$ from the initial 100 fs Gaussian pulse are shuttered and prevented from further amplification, minimizing the chance of a pulse with temporal "wings" being used in the experiment. A Frequency Resolved Optical Gate (FROG)[7] was used to monitor the phase of the pulse on every shot so data could be discarded from a poorly compressed pulses. To suppress ASE, the laser pulse was frequency doubled with a $1.5 \mathrm{~mm}$ thick $\mathrm{KD}^{*} \mathrm{P}$ crystal. Distortions in the pulse profile were controlled by measuring the spectrum and energy of the frequency doubled laser light, then the energy was adjusted until an undistorted frequency spectrum was produced. To check the effects of the ASE, the targets were observed after heating with the unseeded pulse and no damage was observed, suggesting no measurable effect from the focused ASE. A high dynamic range, third order, scanning autocorrelation measurement performed on the $800 \mathrm{~nm}$ pulse suggest the pulse has an intensity contrast of $\mathrm{I}_{\text {background }} / \mathrm{I}_{\text {peak }} \approx 10^{-5}$ at $1.5 \mathrm{ps}$ before the peak of the laser pulse. The contrast is further enhanced (approximately squared) by the frequency doubling. A low dynamic range $\left(10^{-4}\right)$, single shot autocorrelation was performed on every shot and used to monitor the pulse width while an energy calorimeter was used to measure the energy on target. An energy monitor placed behind the target detected no transmitted energy through the foils, suggesting the target remained above the critical density during illumination.

The experiment was conducted with $\hat{S}$ polarized, 150 fs light with a peak intensity of $\approx 2 \times 10^{19} \mathrm{~W} / \mathrm{cm}^{2}\left(I \lambda^{2} \approx 5 \times 10^{18} \mathrm{~W} \mu \mathrm{m}^{2} / \mathrm{cm}^{2}\right)$. The spectroscopic measurements were made with a 500 fs X-ray streak camera interfaced to a dual crystal von Hamös spectrograph (see figure 5). The streak camera fit inside the vacuum chamber, allowing the slit to lie on the focal axis of the crystals. The duel crystal design allowed simultaneous collection of time resolved data from multiple He-like spectral lines on a single shot. Two RbAP crystals were bent to 3.6 and $3.0 \mathrm{~cm}$ to collect time resolved data from the $\mathrm{He}_{\alpha}$ transition and the $\mathrm{He}_{\beta}, \mathrm{He}_{\gamma}$ transitions, respectively. The reflecting surface of the crystals were oriented at approximately $83^{\circ}$ to the target normal, increasing 
the target thickness $t$ along the streak camera viewing cord by approximately $t / \sin \left(17^{\circ}\right)$. The spectral data were focused into the $25.4 \mathrm{~mm}$ long, $150 \mu \mathrm{m}$ wide entrance slit to the streak camera. The high camera sweep speed (2.6 $\mathrm{ps} / \mathrm{mm}$ ) allowed the observation of the transit time difference between the $\mathrm{x}$-rays reflecting off two crystals, producing a spatial gap between the streaked data from the respective crystals. Additionally, the transit time difference of the x-rays of different wavelengths reflected off a single crystal was used to calibrate and check linearity of the sweep for each shot. The streaked data was intensified and collected with a 1024 x 1024 CCD camera.

The X-rays emitted between $10 \mathrm{keV}$ and $1 \mathrm{MeV}$ were collected using a scintillatordiode array. The X-rays with energy above $30 \mathrm{keV}$ were detected using 12 filtered photomultipliers while six filtered $\mathrm{x}$-ray diodes were used for energies below $30 \mathrm{keV}$. These data were electrically relayed to analog to digital converters and stored on a computer. Each foil was suspended over an array of $1.7 \mathrm{~mm}$ diameter holes in a ceramic substrate. The $1.7 \mathrm{~mm}$ diameter foil area was destroyed and replaced after each shot.

\section{Data and Analysis}

An estimate of the optical depth using the expression[8] $\tau(\nu)=\rho \kappa(\nu) \cdot l$ for the conditions $\mathrm{T}_{e}=500 \mathrm{eV}$ and $\mathrm{N}_{e}=1 \times 10^{23} \mathrm{e} / \mathrm{cm}^{3}$ suggest the $\mathrm{He}_{\alpha}$ has an optical depth greater that one for our plasma while the $\mathrm{He}_{\beta}$ and $\mathrm{He}_{\gamma}$ has an optical depth less than one. Thus, the data analysis was divided into two distinct sections:1) analysis of the satellite effect on the spectral lines using the $\mathrm{He}_{\beta}$ and $\mathrm{He}_{\gamma}$ data set and 2) analysis of the spectral opacity effects using the $\mathrm{He}_{\alpha}$ data set.

\section{1 dielectronic satellites}

Spectral data was collected in a single shot. Uncorrected, time resolved data from the $500 \mathrm{Al}, 250 \mathrm{C} / \mathrm{Al}$, and $250 \mathrm{Al} / \mathrm{C}$ foils are shown in figure 5 . These data were collected with a laser intensity $\mathrm{I} \approx 2 \times 10^{19} \mathrm{~W} / \mathrm{cm}^{2}$. Several spectral and temporal features are worth noting. The data shows satellite emission on the long wavelength side of $\mathrm{He}_{\alpha}$ (1s2lnl' manifold) and between the $\mathrm{He}_{\beta}$ and $\mathrm{He}_{\gamma}$ transitions (Li-like and Be-like satellite manifolds). The satellite emission increases in number and intensity with amount of aluminum present in the target. In general, the time history of the satellites is fairly short ( $\leq 3 p s$ FWHM) where as $\mathrm{He}_{\alpha}$ tends to be relatively long ( $\geq 7$ ps FWHM). The higher n-state resonance lines $\left(\mathrm{He}_{\beta}\right.$ and $\left.\mathrm{He}_{\gamma}\right)$ also tend to be longer than the satellite emission, although not nearly so long. 
Finally, the so-called Li-like "blue" satellite appears in the data. Although it is not used in this analysis, it is interesting to note several characteristics of this feature. This satellite has been attributed to the $1 s 2 p\left[{ }^{1} P\right] 3 d^{2} D_{5 / 2}-$ $1 s^{2} 3 d^{2} D_{5 / 2}$ and $1 s 2 p\left[{ }^{1} P\right] 3 d^{2} D_{3 / 2}-1 s^{2} 3 d^{2} D_{3 / 2}$ transitions [9]. The time history is noted as distinctly short lived $(\approx 1 p s)$, suggesting conditions suitable for its production being extremely transient in nature.

To analyze the data, corrections were made for the x-ray transit time difference, the photocathode response, the wavelength dependent solid angle, and the crystal reflectivity.

\subsubsection{Satellite Modeling}

The satellite data reduction focused on the spectral region between the $\mathrm{He}_{\beta}$ and $\mathrm{He}_{\gamma}$ transitions. The spectral data was modeled by calculating the atomic structure and cross-sections for a set of plasma conditions (density and temperature). These data are input into a non-LTE, collisional-radiative model to generate simulated spectra, then a comparison is made to a temporal slice from the experimental data record. The process is repeated until a good agreement between experiment and simulation is found

As can be seen in the data, a near continuum of satellites features are produce between the $\mathrm{He}_{\beta}$ and $\mathrm{He}_{\gamma}$ transitions. Including all the necessary ions and levels quickly adds and makes a calculation of the level populations time consuming. Thus, the level populations were calculated using two methods; detailed configuration modeling and configuration averaged (CA) modeling. The detailed calculation was used to benchmark the configuration averaged model. When the benchmark was shown to be accurate, the configuration averaged model was used to generate synthetic spectra. The detailed spectral modeling was performed using the Hebrew University Lawrence Livermore Atomic physics Code (HULLAC) to calculate the atomic structure and cross-sections. A detailed description of the HULLAC code is given in reference[10]. HULLAC includes the physical processes of collisional excitation and de-excitation, collisional ionization, radiative decay, autoionization, dielectronic, three-body, and radiative recombination. The $\mathrm{H}$-like, He-like, Li-like, Be-like, and B-like ions were included in the simulations. A typical HULLAC simulation for $N_{e}=3 \times 10^{23} \mathrm{e} / \mathrm{cm}^{3}$ and $T_{e}=200 \mathrm{eV}$ is shown in figure 5 . For theses simulations, levels $1 \operatorname{snln}^{\prime} l^{\prime}\left(n, n^{\prime} \leq 4\right)$ are included. The simulation shows large Li-like and Be-like satellite contributions between the $\mathrm{He}_{\beta}$ and $\mathrm{He}_{\gamma}$ transitions. With the addition of the $n=5$ level, further contributions appear from B-like satellites.

The CA calculation was performed using the code AVERROĖS. A detailed explanation of AVERROÈS is in reference $[11,12]$. In general, AVERROÈS 
forms "super levels" or superconfigurations from groups of configurations. The superconfigurations are made of subshells that are grouped in "supershells" with an interger number of electrons. The level spacing within the individual groups are close enough to assume LTE statistics. AVERROÈS includes the same physical processes as HULLAC (collisional excitation and de-excitation, collisional ionization, etc.). With the data from AVERROĖS as input, the code TRANSPEC $[13,14]$ was used to generate the NLTE level populations and synthetic spectra. TRANSPEC is a 1-D, time dependent, collisional-radiative model that self-consistantly couples atomic population data with radiation. The hydrodynamics code LASNEX[15] was also used and showed similar results. A comparison of HULLAC and AVERROĖS is shown in figure 5. The AVERROES approximation to the detailed HULLAC model is shown as an overlay in the figure. The 1-D conditions were obtained from the hydrodynamics code FILM[16]. The plasma conditions of the central cell are shown in figure 5. Using AVERROES and TRANSPEC, synthetic spectra were produced for each foil thickness. The data and synthetic spectra are displayed side by side in see figures 5 . In general, the agreement is fairly good. Early in time, the results suggest an ambiguous seperation between the resonance lines and the dielectronic satellites. After roughly two picoseconds, the satellites subside and the resonance lines begin to dominate the line centroid. Although this study was performed at $10^{19} \mathrm{~W} / \mathrm{cm}^{2}$, this satellite production has been observed at lower laser intensities as well[17]. Because of the extensive modeling required to extract the satellites from the resonance lines (i.e., the need to include such a large number of levels to accurately model the line shapes) it is unclear that line shifts can be extracted from these plasmas.

\section{2 line center opacity}

In addition to the numerous satellites affecting the line width, the opacity will also broaden the line shape. For the experiment considered here, the line center opacity of the $\mathrm{He}_{\alpha}, \mathrm{He}_{\beta}$, and $\mathrm{He}_{\gamma}$ transitions is considered. The line center opacity can be approximated using the expression[8],

$$
\tau(\omega) \approx 2 \pi^{2} r_{0} c f_{b a} N_{1} L_{a}(\omega) \frac{l}{\mu}
$$

where $r_{0}$ is the classical electron radius, $f_{b a}$ is the absorption oscillator strength, $L_{a}(\omega)$ is the line shape, $N_{1}$ is the ground state concentration, $l$ is the the target thickness and $\mu$ is the angle between the target normal and the viewing line on sight. Here, the code Line-2T[18] was used to estimate the line shapes. Even for large concenstrations of He-like $\left(N_{H e-l i k e} / N_{\text {total }} \geq 80 \%\right)$, the $\mathrm{He}_{\beta}$ and $\mathrm{He}_{\gamma}$ transitions are optically thin and thus experience little effect 
Table 1

Table showing percentage increase in line shape with increasing opacity

\begin{tabular}{|c|c|c|} 
time (ps) & \% change-250 C/Al & \% change-250 Al/C \\
\hline 0.0 & 40.4 & 10.1 \\
2.0 & 36.4 & 9.3 \\
4.7 & 19.1 & 4.0 \\
7.0 & 10.1 & 0.15
\end{tabular}

due to opacity. The line center opacity of the $\mathrm{He}_{\alpha}$ transition is greater than one when the concentration of He-like ions is greater than $5 \%$.

The line center opacity of the $\mathrm{He}_{\alpha}$ transition in these experiments was determined by finding a multiplier so that the satellite intensities of the $250 \mathrm{C} / \mathrm{Al}$ foil and $250 \mathrm{Al} / \mathrm{C}$ foil match the satellite intensity of the $500 \mathrm{Al}$ foil. Because the satellite emission should be optically thin in all cases and the conditions are assumed to be the same for all foils, the multiplier is applied to the entire spectrum and the line center of the $\mathrm{He}_{\alpha}$ transition is compared. With the multiplier $\approx 2$, the comparison of the line center intensities yield,

$$
\frac{2 I_{1}}{I_{2}} \approx \frac{S_{1}\left(1-\exp \left(\frac{\tau}{\mu}\right)\right)}{S_{2}\left(1-\exp \left(\frac{2 \tau}{\mu}\right)\right)}
$$

where $S_{1}$ and $S_{2}$ are the relative source functions. A sample of the comparison at 2 ps after the peak of the pulse is shown in figure 5. It is worth noting that the opacity value $\tau$ produced by comparing the $250 \mathrm{C} / \mathrm{Al}$ foil to the $500 \mathrm{Al}$ foil is different from that produced by comparing the $250 \mathrm{Al} / \mathrm{C}$ foil to the 500 Al foil. The implications of this will be addressed in a future work. Here, we consider opacity both cases and observe the effect on the $\mathrm{He}_{\alpha}$ transition. A plot of the inferred opacity $\tau$ as a function of time is shown in figure 5.

To analyze the effects on the range of opacities have on the spectral line width, we assume the simpliest case where the line shape is Gaussian. The half-widthhalf-maximum as a function of the line center opacity can be expressed as[19],

$$
x_{H W H M}=\left[\ln \left(\tau_{0}\right)-\ln \left[-\ln (0.5)-\ln \left(1+\exp \left(-\tau_{0}\right)\right)\right]\right]^{1 / 2}
$$

where $x_{H W H M} \equiv\left(\nu-\nu_{0}\right) / \Delta \nu_{D}, \nu_{0}$ is the line center frequency, $\Delta \nu$ is the Doppler HW $(1 / e) \mathrm{M}$ halfwidth, and $\tau_{0}$ is the line center opacity. Using this estimate, the correction to the line width for the conditions in our experiment is shown in table 1.

Early in time, the effect is somewhat severe, particularly when using the opacity derived from the $250 \mathrm{C} / \mathrm{Al}-500 \mathrm{Al}$ foil comparison. It is always less severe 
when using the opacity derived from the $250 \mathrm{Al} / \mathrm{C}-500 \mathrm{Al}$ foil comparison. The average of the two suggest a maximum $25 \%$ effect on the line shape for these conditions. In general, this effect is expected to be less than the effect of the satellites on the line shape.

\section{Conclusions}

We have performed an experiment to observe satellite and opacity effects on the the $\mathrm{He}_{\alpha}, \mathrm{He}_{3}$, and $\mathrm{He}_{\gamma}$ transitions in ultrashort pulse laser heated aluminum foils at an intensity of $1 \times 10^{19} \mathrm{~W} / \mathrm{cm}^{2}$. We observed a significant contirbution from the satellites on all three resonance lines at times $t \leq 3 \mathrm{ps}$. The satellite effect on the resonance lines decrease rapidly. The opacity broadening on the $\mathrm{He}_{\beta}$, and $\mathrm{He}_{\gamma}$ transitions is neglectiable. The opacity broadening on the $\mathrm{He}_{\alpha}$ transition is estimated to be a maximum of $40 \%$ (early in the emission) and in general, much less than the contribution from the satellite emission.

\section{Acknowledgment}

The authors are grateful for useful discussions with Drs. Carlos Iglesias and Brian Wilson. This work was performed under the auspices of the U.S. Department of Energy by University of California Lawrence Livermore National Laboratory under contract No. W-7405-Eng-48.

\section{References}

[1] S. Volonte, Polarisation shift effect in high-density plasmas., Journal of Physics D (Applied Physics) 11 (12) (1978) 1615.

[2] B. Henry, Spectral line limiting and polarization shift in plasmas of high particle and energy density., Lasers and Particle Beams 1 (1) (1983) 11.

[3] Y. Xin-Zhong, S. Ichimaru, Theory of interparticle correlations in dense, high temperature plasma. VII. polarization shift of spectral lines., Phys. Rev. A 34 (3) (1986) 2173.

[4] S. Boddeker, S. Gunter, A. Konies, L. Hitzschke, H. J. Kunze, Shift and width of the $\mathrm{H}_{\alpha}$ line of hydrogen in dense plasmas., Phys. Rev. E 47 (4) (1993) 2785.

[5] U. Andiel, K. Eidmann, K. Witte, I. Uschmann, E. Forster, Comparative study of time-resolved $\mathrm{K}$-shell spectra from aluminum plasmas generated by ultrashort laser pulses at 395 and 790 nm., App. Phys. Lett. 80 (2) (2002) 489. 
[6] A. Sullivan, J. Bonlie, D. F. Price, W. E. White, 1.1-J, 120-fs laser system based on Nd:glass-pumped Ti:sapphire., Optics lett. 21 (8) (1996) 603.

[7] D. J. Kane, R. Trebino, Characterization of arbitrary femtosecond pulses using frequency-resolved optical gating, IEEE Journal of Quantum Electronics 29 (2) (1993) 571.

[8] H. Griem, Plasma Spectroscopy, McGraw-Hill Book Company, New York, 1964.

[9] F. B. Rosmej, J. Abdallah, Blue satellite structure near $\mathrm{He}_{\alpha}$ and $\mathrm{He}_{\beta}$ and redistribution of level populations., Phys. Letters A 245 (6) (1998) 548.

[10] A. Bar-Shalom, M. Klapisch, J. Oreg, HULLAC, an integrated computer package for atomic processes in plasmas, J. of Spec. and Rad. Trans. 71 (26) (2001) 169 .

[11] O. Peyrusse, Atomic configuration averages and non-local thermodynamical equilibrium plasma spectroscopy calculations., J. Phys. B 32 (3) (1999) 169.

[12] O. Peyrusse, On the super configuration approach to model NLTE plasma emission, J. of Spec. and Rad. Trans. 71 (2-6) (2001) 571.

[13] O. Peyrusse, A model for the simulation of nonequilibrium line transfer in laboratory plasmas., Phys. of Fluids B 4 (7) (1992) 2007.

[14] O. Peyrusse, X-ray spectroscopy studies using the TRANSPEC code., J. of Spec. and Rad. Trans. 51 (1-2) (2001) 281.

[15] G. Zimmerman, D.Kershaw, D. Bailey, J. Harte, The LASNEX code for inertial confinement fusion., in: Topical Meeting on Inertial Confinement Fusion, San Diego, CA, USA, 7-9 Feb. 1978., Vol. 1-4, O.S.A., Optical Society of America, 2010 Massachusetts Ave., NW, Washington, DC 20036, USA, 1977, p. 700.

[16] U. Teubner, P. Gibbon, E. Forster, F. Fallies, P. Audebert, J. Geindre, J. Gauthier, Subpicosecond $\mathrm{KrF}^{*}$-laser plasma interaction at intensities between $10^{14}$ and $10^{17} \mathrm{~W} / \mathrm{cm}^{2}$, Phys. of Plas. 3 (7) (1996) 2679.

[17] A. M. Urnov, A. Y. Faenov, T. A. Pikuz, I. Y. Skobelev, J. Abdallah, R. E. H. Clark, J. Cohen, R. P. Johnson, G. A. Kyrala, A. L. Osterheld, X-ray spectra of multiply-charged hollow ions in the emission from a femtosecond laser plasma., JETP Letters 67 (7) (1998) 489.

[18] C. A. Iglesias, F. J. Rogers, R. Shepherd, A. Bar-Shalom, M. S. Murillo, D. P. Kilcrease, A. Calisti, R. W. Lee, Fast electric microfield distribution calculations in extreme matter conditions., Quant. Spect. and Rad. Trans. 65 (1-3) (2000) 303.

[19] S. O. Kastner, A. K. Bhatia, Half-widths, escape probabilities and intensity factors of opacity-broadened Doppler and Voigt-profile lines., Quant. Spect. and Rad. Trans. 58 (2) (1997) 217. 
Fig. 1. Experimental setup showing target, streak camera, and duel crystal von Hamös spectrograph

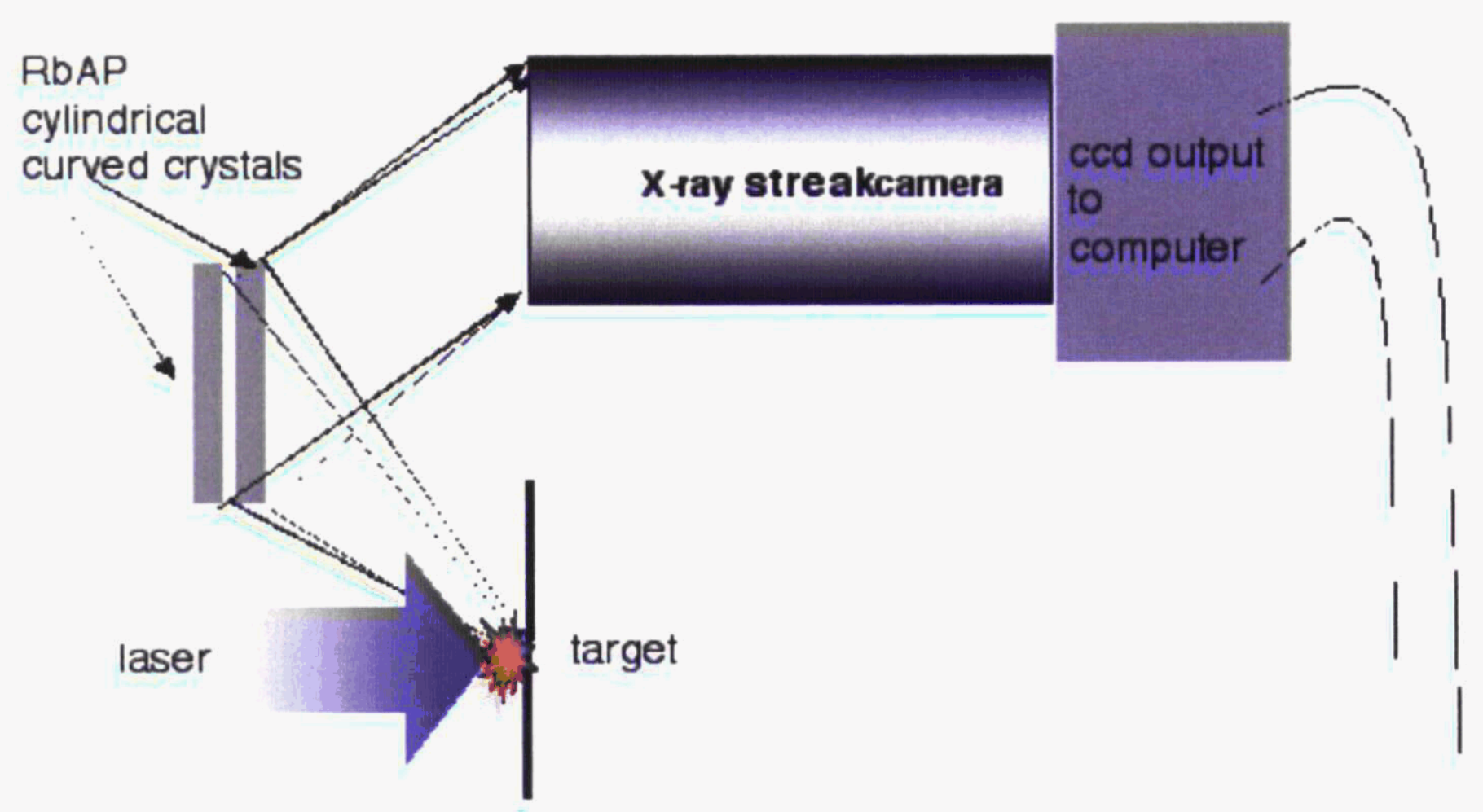



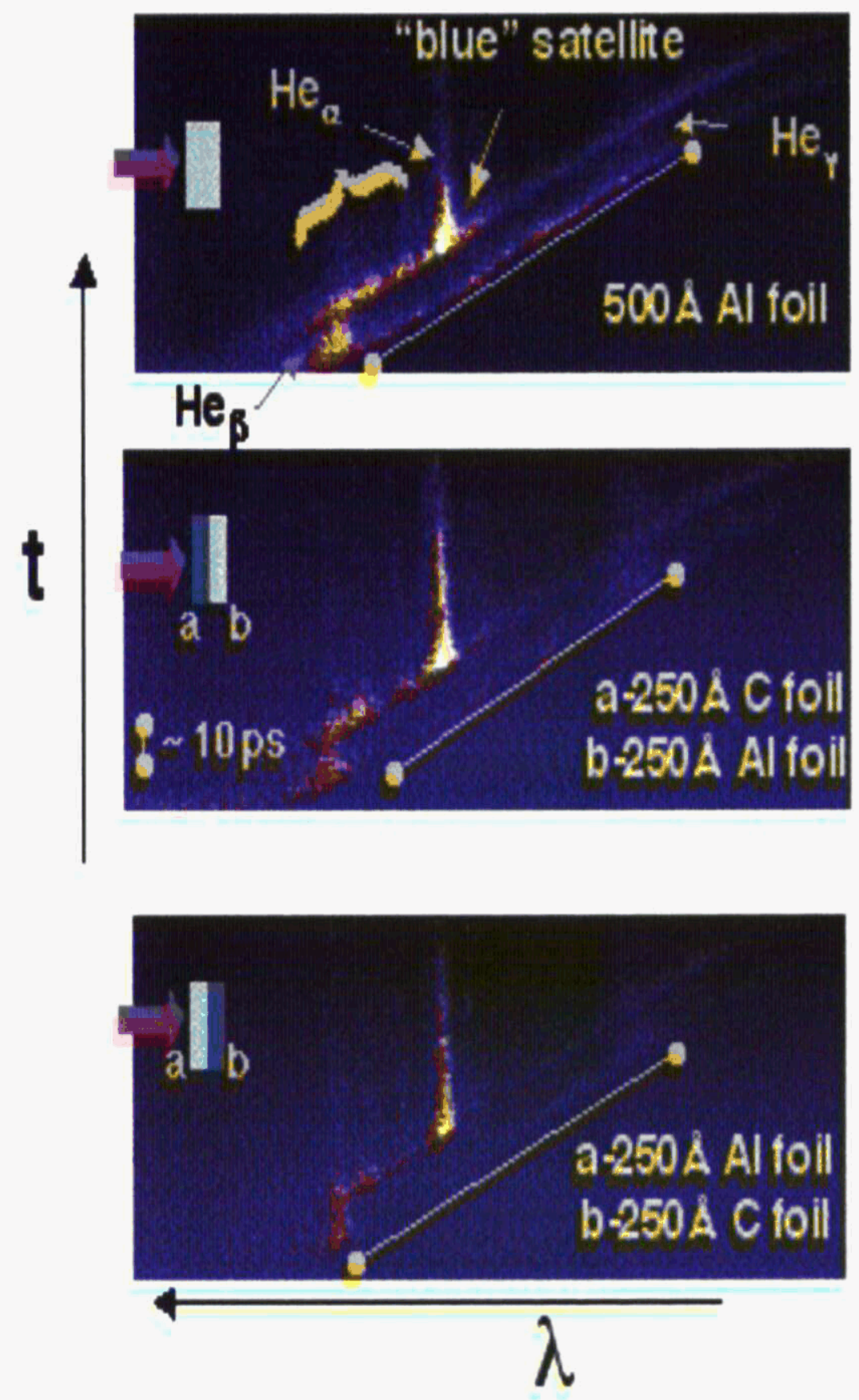

Fig. 2. Data from $250 \mathrm{C} / \mathrm{Al}, 250 \mathrm{Al} / \mathrm{C}$, and $500 \AA$ thick aluminum foils 
Fig. 3. Comparison between AVERROÈS/TRANSPEC (solid line) and HULLAC (vertical lines) for $N_{e}=3 \times 10^{23} \mathrm{e} / \mathrm{cm}^{3}$ and $T_{e}=200 \mathrm{eV}$.

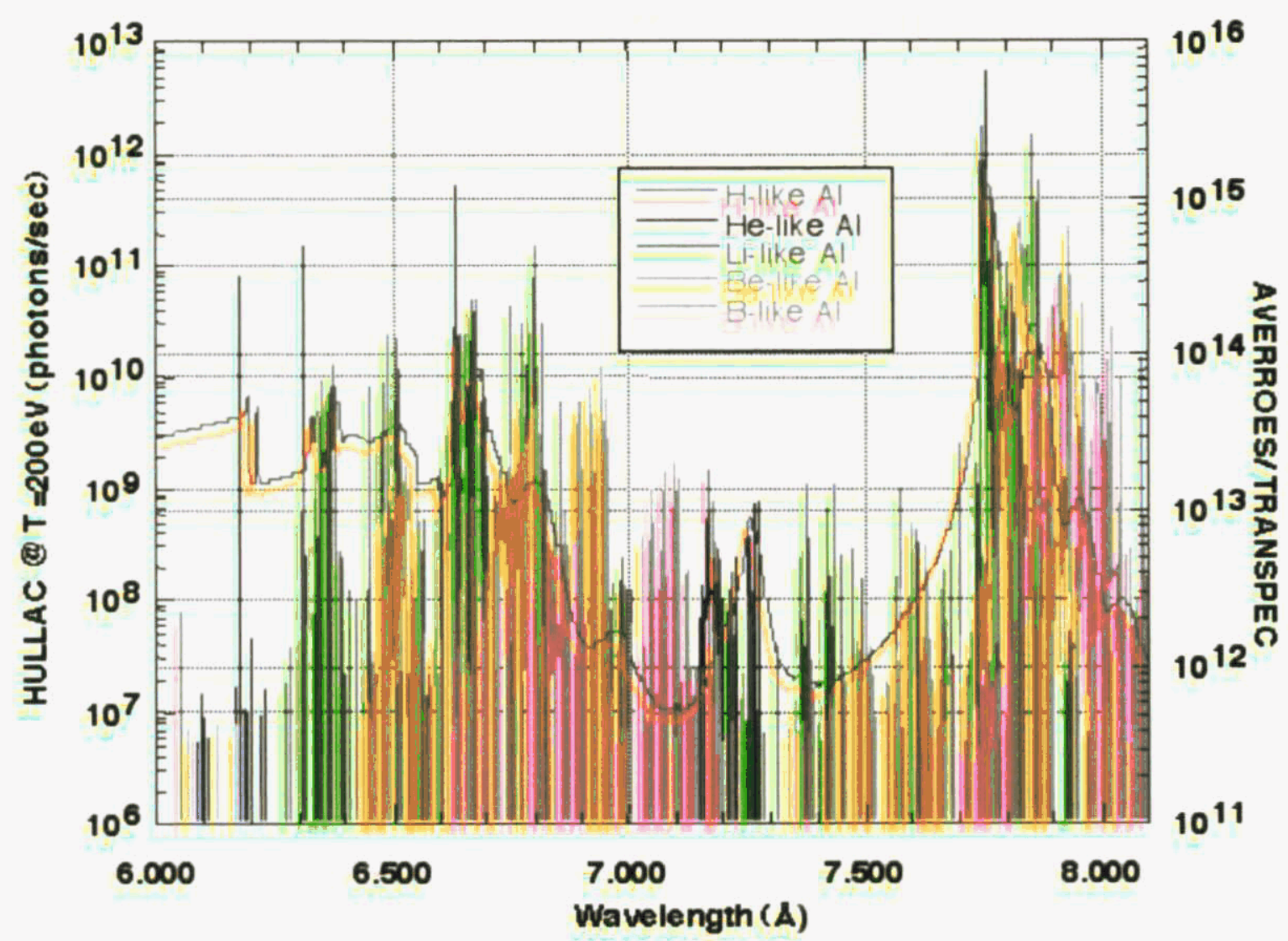


Fig. 4. Hydrodynamics simulation of a $500 \AA$ foil $(100$ fs pulse, $\left.I \lambda^{2}=5 \times 10^{18} \mathrm{~W} / \mathrm{cm}^{2} \mu \mathrm{m}\right)$

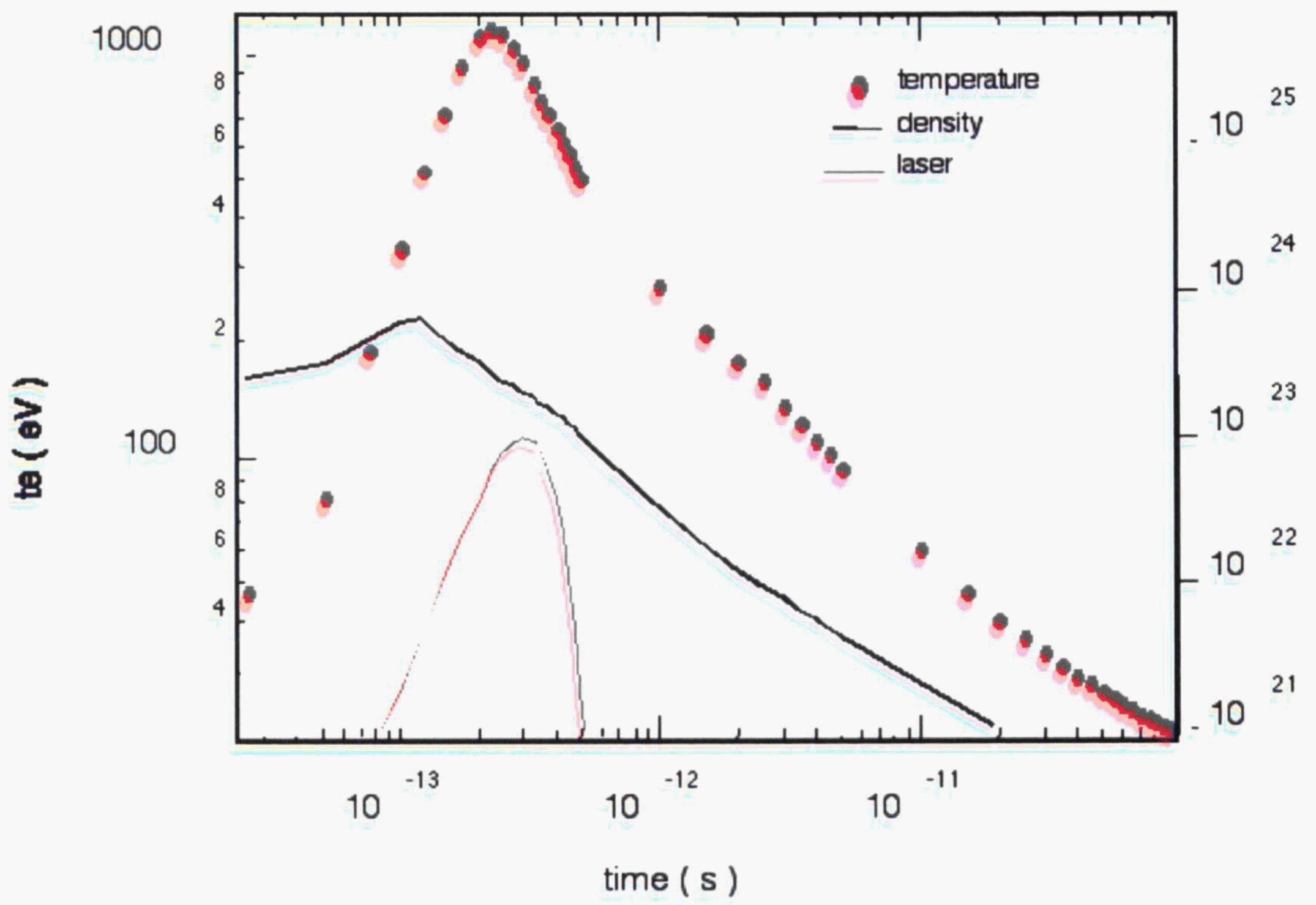



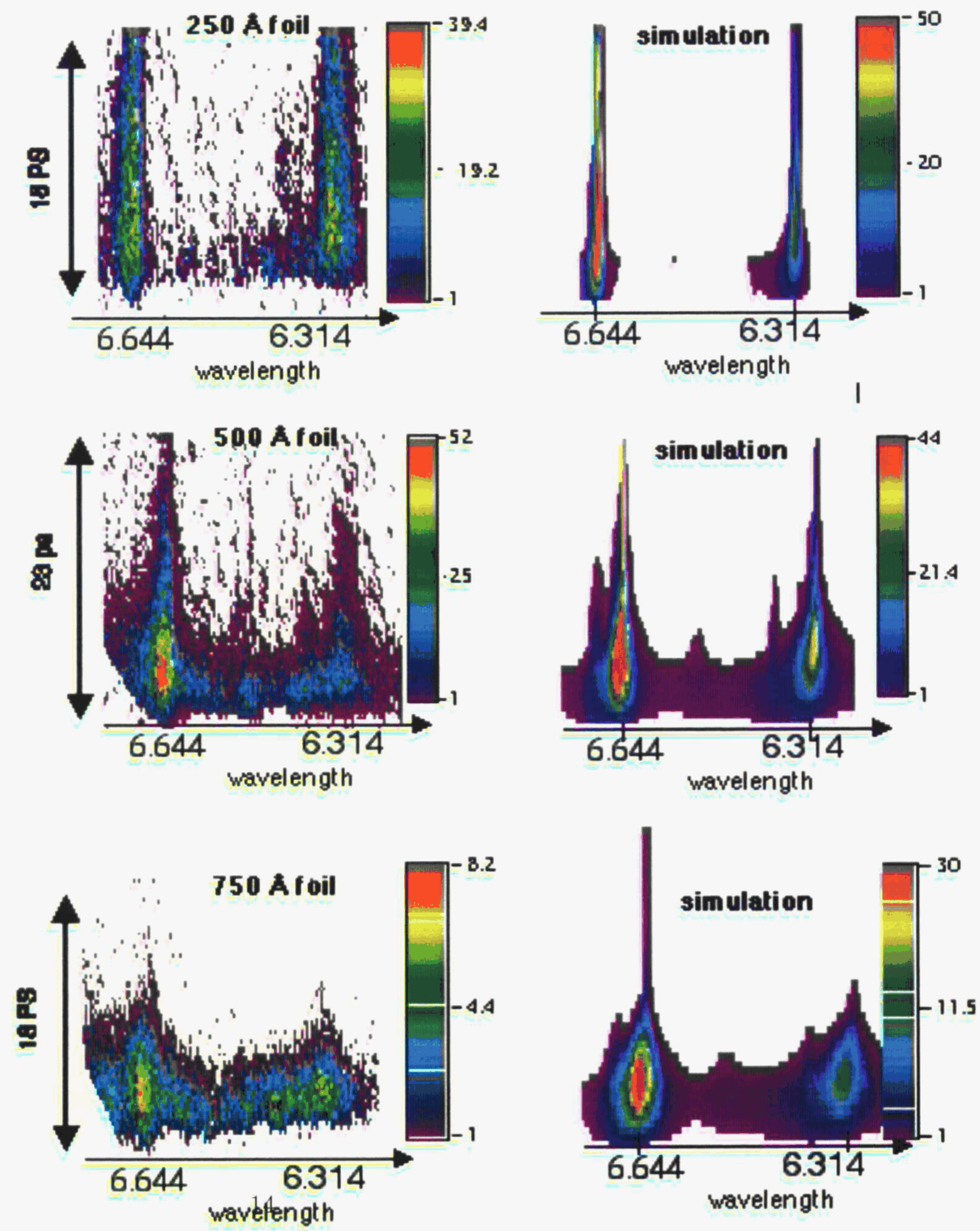
Fig. 6. Comparison of $\mathrm{He}_{\alpha}$ emission from the $250 \mathrm{C} / \mathrm{Al}$ foil, the $250 \mathrm{Al} / \mathrm{C}$ foil, and the $500 \mathrm{Al}$ foil after matching the Li-like satellite intensities from the three foils.

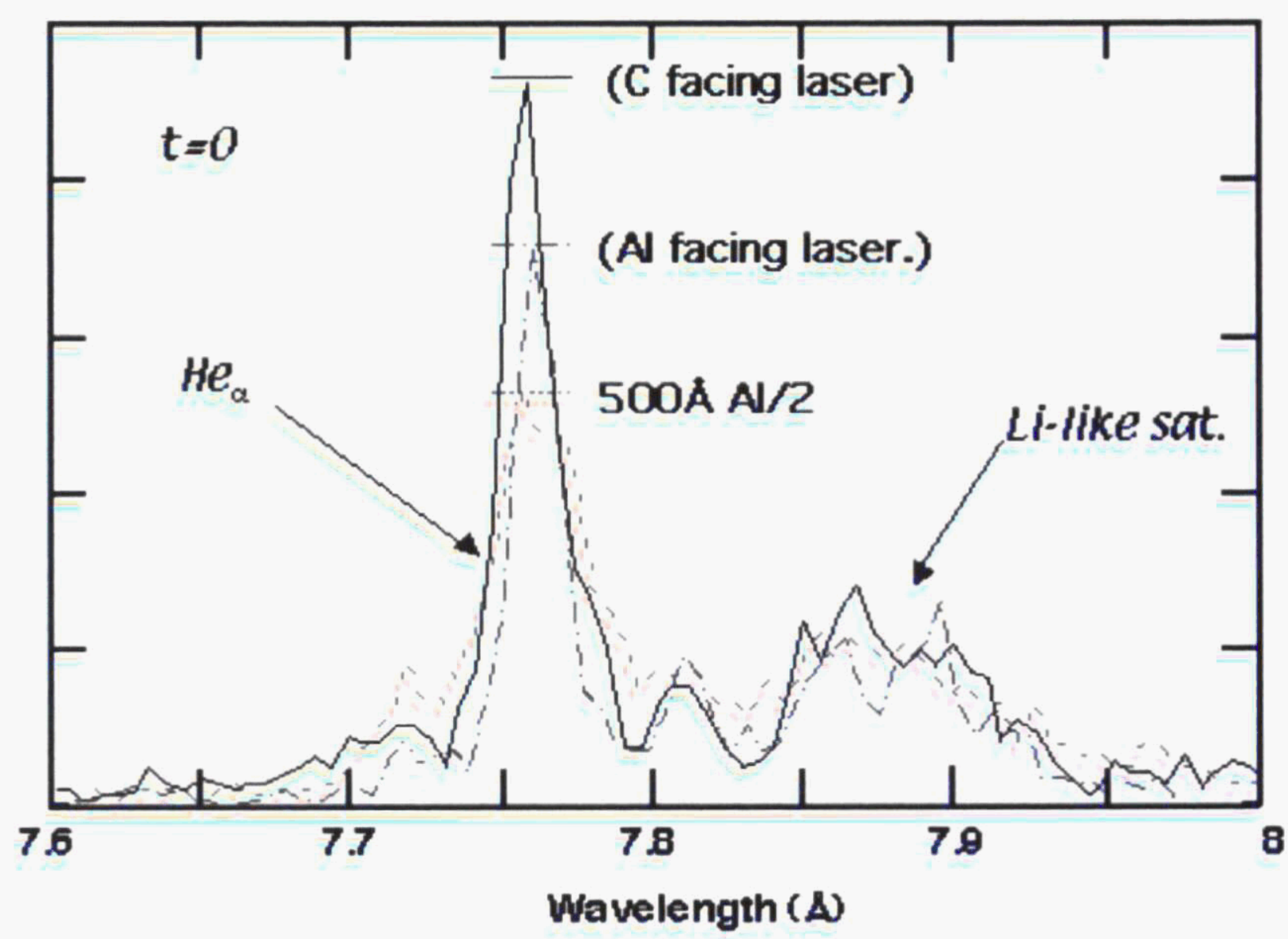


Fig. 7. The time dependent opacity of the $500 \mathrm{Al}$ foil as determined by using the $250 \mathrm{C} / \mathrm{Al}$ and $250 \mathrm{C} / \mathrm{Al}$ foils

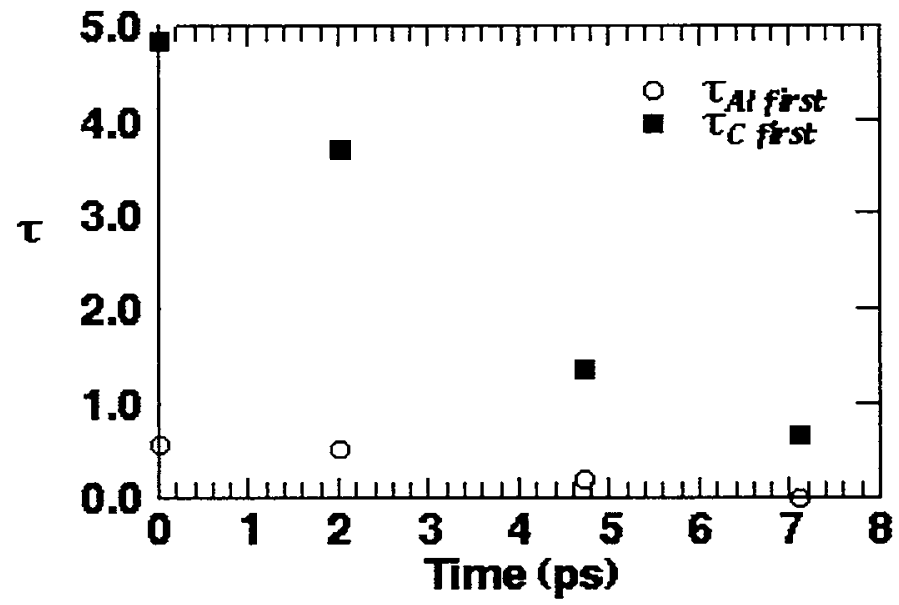

